\title{
Klaus Ehringfeld Multikulturalität im deutschen internationalen Familienrecht
}

\begin{abstract}
Der Begriff "multikulturelle Gesellschaft« bezeichnet den Tatbestand, daß die Bundesrepublik in den vergangenen Jabrzehnten zu einem Einwandererland geworden ist. Die soziale Integration unterschiedlicher ethnischer Gruppen wirft eine Fülle ungelöster Probleme auf, für die jedoch eine Reihe von Modellen zur Diskussion stehen. Die rechtliche Problematik, inwieweit Rechtsordnungen anderer Länder, insbesondere islamischer und afrikanischer, in der Bundesrepublik Anwendung finden, wird bislang jedoch nur ansatzweise diskutiert. Mit der Frage der Abgrenzung ausländischer und deutscher Familienrechtsnormen beschäftigt sich der Aufsatz von Ehringfeld, der für eine zweistufige Lösung plädiert: zunächst ist die Frage der anwendbaren Rechtsordnung zu klären, sodann die das Einsatzes des ordre public zu erörtern. Der Autor diskutiert die These, ausländische Familien nur auf einen elementaren Grundrechtsbereich unrelativierbar zu verpflichten, während ibnen in einem peripheren Raum gestattet wird, ibre Verbältnisse grundrechtsautonom zu gestalten.
\end{abstract}

Die Red.

\section{Einleitung}

Deutschland hat sich in den vergangenen knapp i so Jahren von einem der ersten und größten Auswanderungsländer zu einem der bedeutendsten Einwanderungsländer entwickelt. Ende 1994 lebten annähernd sicben Millionen Menschen mit ausländischem Paß in der Bundesrepublik. ${ }^{1}$ Neben Arbeitsmigranten und deren Nachfahren setzt sich diesc Zahl zunehmend aus Flüchtlingen aller Kontinente zusammen, die durch den Zerfall des sowjetischen Imperiums und die Renaissance des Denkens in nationalen Kategorien, durch weltwirtschaftliche Umstrukturierungen, ökologische Zerstörung sowie demographisches Wachstum dazu gezwwungen wurden, ihre Heimat zu verlassen. ${ }^{2}$ Diese Migrationsprozesse haben die gesellschaftliche Struktur der Bundesrepublik verändert.

In ciner bis dahin relativ homogenen Nationalgesellschaft haben sich Einwanderungsminderheiten herausgebildet, deren sprachliche, ethnische und kulturelle Identität von derjenigen der autochthonen Bevölkerung erhcblich abweicht. ${ }^{3}$ Dabei ist

1 Bericht der Auslanderbeauftragten uber die Lage der Auslander in der Bundesrepublik Deuschland (1995), S. 12.

2 Bade (Hrsg.), Das Manifest der 60 (1994), S. 109.

3 Perout, Europas Zukunft: mulukulturell oder interkuliurell?, in: Die Neue Gesellschaft/trankfurter Hefte (1989), S. 536 f.; Habermas, Anerkennungsliampfe im demokratuschen Rechtsstaat, in: Taylor, Mulrikulturalısmus und die Politık der Anerkennung (1993), S. 171; Lepenies, Die Übersetzbarkeit ditr Kulturen - Ein europaisches Problem, eine Chance fur Europa (unveroffentlichtes Manuskripr 1973) S. 21, bezeichnet dieses Phanomen als Inselbildung. Es hatten sich ungeplant und vor allem ungewollt -Exklaven der Drtuten Welt in der Ersten* ausgebildet. 
ein sozialer Prozeß ausgelöst worden, den alle Beteiligten ursprünglich nicht intendiert hatten - die dauerhafte Niederlassung von ethnischen Minderheiten aus politisch und ökonomisch instabilen Ländern, insbesondere auch Entwicklungsländern, in den wirtschaftlich erfolgreichen und rechtsstaatlichen Wohlfahrtsstaaten Zentralund Nordeuropas. ${ }^{4}$ Diese Entwicklung erscheint heute irreversibel; vielmehr ist $\mathrm{zu}$ erwarten, daß sich dieses Phänomen in Zukunft eher verfestigen als auflösen wird.'

Mit der dauerhaften Niederlassung ist in den Aufnahmeländern, so auch in der Bundesrepublik, ein Prozeß der ethnischen Pluralisierung und kulturellen Diversifizierung sowie der geographischen Konzentration bestimmter Volksgruppen in ethnisch relativ homogenen Wohngebieten einhergegangen, dessen Resultat mit dem Begriff multikulturelle Gesellschaft bezeichnet wird. Um diesen Begriff rankt sich die aktuelle Diskussion zum Umgang mit ethnischen Minoritäten. Von einigen nur als deskriptive Kategorie verstanden, verkörpert sie für ihre Gegner die Büchse der Pandora, durch welche die kulturelle deutsche Identität sowie die politischen, rechtlichen und sozialen Grundfesten der Gesellschaft in Gefahr gebracht werden. ${ }^{6}$ Ihre Befürworter hingegen interpretieren sie als normative Herausforderung und sehen mit ihr eine Gesellschaft skizziert, in der ausländerfeindliche Verhaltensweisen unc Einstellungen überwunden sind und ein gleichberechtigtes Leben verschiedener Ethnien und Nationalitäten möglich ist. ${ }^{?}$

Die gesellschaftliche Entwicklung hat eine Viclzahl ungelöster rechtlicher Fragen manifest werden lassen. ${ }^{8} \mathrm{Im}$ besonderen Maße stellen sie sich im Familienrecht als demjenigen Rechtsgebiet, das von Traditionen, Religionen und Ideologien am stärksten durchdrungen ist, das am deutlichsten die kulturellen Wurzeln eines Landes reflektiert. Dem entspricht es, daß das Familienrecht gerade in den vergangenen Jahren an Aufmerksamkeit gewonnen hat, was sich an einer steigenden Zahl von familienrechtlichen Streitigkeiten mit Auslandsberührung ausdrückt. ${ }^{9}$

4 So wohl als erster Zuleeg, DVBI. 1974, 34 I, 347 (... ist die Bundesrepublik fakusch ein Einwanderungsland); vgl. auch Oberadorfer, ZAR 1989,3 , 10 (... derzeit größten Lebensluge der deutschen Politik: "Diè Bundesrepublik Deutschland ist kein Einwanderungsland. . s); Bade (Fon. 2), S. 214 ; aus konservatuver Sicht siche nur Quaritsch, Einwanderungsland Bundesrepublik Deutschland (198:), S. 12 (Die Leuģnung det Einwanderung...).

s Diese Annahmen stützen zum einen Bcrechnungen des Bundesinstituts fur Bevołkerungsforschung (BIB). Danach wird es im Jahre 2040 ungefahr zwolf Millionen Zuwanderer in der Bundesrepublik geben, fas doppelt soviel wie gegenwartig (rund sieben Millionen), vgl. Sudde:tsche Zettung vom 6. 9. 1994. Daruber hinaus ist zu erwarten, daß ethnische Vertreibungen in der Gegenwart und nahen Zukunft als Wanderungsmotiv zunehmen werden.

$6 \mathrm{Vgl}$. hierzu nur das sHeidelberger Manifest $\alpha$ vom 17.6. 1981 (abgedruckt in der Frankfurter Rundschas vom 4.3.1982, S. 14), in dern acht bundesdeutsche Hochschullehrer vor ciner "Unterwandcrung des Deutschen Volkes durch Zuzug von Millionen von Auslandern w wamen, die zu sbekannten erhnischen Katastrophen multıkultureller Gesellschaften- führen wurde; vgl. auch den Artikel des (damalıgen) bayerischen Staatsministers Edmund Stoiber, in: Die Welt vom 25.2. 1989, S. 17 (Und deutsche Richter blattern im Koran), fur den das *Gerede von der multikulturellen Gesellschaft... die Axt an die Wurzeln unserer in Jahrhunderten entwickelten nationalen und kulturellen Identitats legt. Vgl. des weiteren den Beitrag von Eckart Schiffer, Leiter der Verfassungsabtelung bcim Bundesinnenminister und „Chefdenker* der Ausländerpolitik, in: Der Sptegel, Heft 40/199s, S. 53-59. ("Der Koran ist nicht Gesetz*), der gegen eine smultikulturelle Mosaikgesellschaft « argumentiert.

7 Vgl. die gute Übersicht bei Schulte, Multıkulturelle Gesellschaft - Chance, Ideologse oder Bedrohung?, In: aus Politik und Zeitgeschichte, Beilage zur Wochenzertschnft "Das Parlament «, Juni 1990 B $23-24$, S. 3 , sti., 9 ff.

8 Exemplarısch seien nur die ungeklarten Voraussetzungen einer erleichterten Eınburgerung der Auslander oder die Zubilligung eines Wahlrechts auf kommunaler Ehene fur die Migranten genannt, vgl. zum letz.teren auch BVerfGE 83, $37=$ BVerfG NJW 1991, 162

9 An dieser Stelle sei nur auf die annahernd 60000 Ehen verwiesen, die 1993 von Migranten in der Bundesrepublik geschlossen wurden. Fast socoo dieser Verbındungen waren solche zwischen Deutschen und Ausländern. Gerade bei diesen ist die Scheidungsrate besonders hoch, vgl. Statistisches Bundesamt (Hrsg.), Im Blickpunkt: A uslandische Bevolkerung in Deutschland (1995), S. 26 sowie Staudinger/Coester $\$ 1671 \mathrm{Rn} .228$ 
Fragen von eminenter sozialer und rechtlicher Bedeutung stellen sich dabei vor allem in dem Moment, in dem darüber zu befinden ist, wie weit in einer Einwanderungsgesellschaft wie der deutschen Toleranz gegenüber Rechtcn geübt werden kann, welche die Migranten aus der Heimat mitgebracht haben, und inwieweit diese Heimatrechtsverbundenheit zurückgedrängt werden kann. Es geht dabei letztlich um die Durchsetzungsfähigkeit fremder Rechts- und Moralvorstellungen in der Bundesrepublik im Einzelfall.

Besonders konflikttragend sind hierbei die Rechtsnormen aus dem islamischen ${ }^{\circ}$ und dem afrikanischen Rechtskreis, die sich stark von westeuropäischen Rechtsordnungen unterscheiden. Hier ist die Diskrepanz zum deutschen Familienrecht besonders groß, dies gilt insbesondere nach den Reformen des deutschen materiellen Familienrechts." So erscheint es als ein zwingendes Desiderat, Strukturen aufzudecken und Normen zu entwickeln, welche die Grenzen abstecken, jenseits derer uniforme Lebensmuster auch von Migranten in der Bundesrepublik gefordert werden müssen.

\section{Der Widerspruch zwischen sozialer und rechtlicher Entwicklung}

Innerhalb dieser familienrechtlichen Fragen zur Abgrenzung ausländischer und deutscher Vorstellungen von Rechts- und Wertesystemen widmen die Gerichte der Regulierung von Eltern-Kind-Konflikten in Migrantenfamilien erhöhte Aufmerksamkeit. Inhaltlich geht es darum, nach welchem Recht familiärc Konflikte bei der Ausübung des Sorgerechts (vgl. $\$ 1666$ BGB) und um die Verteilung der elterlichen Sorge bei Scheidung oder Getrenntleben (vgl. $\$ \$ 1671,1672$ BGB) geregelt werden und wieweit das gegebenenfalls anzuwendende fremde Recht akzeptiert werden kann. ${ }^{12}$

Ein Blick auf die Entwicklung der Rechtsprechung zeigt, daß die Gerichte zunehmend eine eingriffsfreudige Position einnehmen und das ausländische Recht immer öfter und umfassender von der Anwendung in der Bundesrepublik ausschließen. Hielten sie sich vor 25 Jahren bei Interventionen in Migrantenfamilien noch stark zurück und ließen das fremde Recht fast ausnahmslos durchgreifen, ${ }^{13}$ so wird zur Regulierung der Konflikte zunehmend die lex fori herangezogen. Dies geschieht entweder über die entsprechende Intcrpretation der einschlägigen Abkommen, hier vor allem des Haager Minderjährigenschutzabkommens (MSA), ${ }^{14}$ oder über den ordre public aus Art. $6 \mathrm{EGBGB}$, "s der als ultima ratio die an sich gebotene Anwendung

10 Dies ist zum einen mit der zahlenmäßigen Bedeutung der islamischen Gemeinde in Europa im allgemeinen und in Deutschland im besonderen zu erklaren. Von den 20 Millionen Moslems in Europa leben zwolf Millionen als Migranten in Westeuropa, rund zwei Millionen davon in Deutschland, vgl. dazu Tibi, Im Schatten Allahs (1994), S. 177; Cavdar, RdJB 1993, 265. Zum anderen hangt es damit zusammen, daß sich das islamische Recht durch Vorstellungen von Kindes- und Elternrechten auszeichnet, die westeuropaischen Ansichten oftmals sehr fremd sind.

11 Vgl. hier nur das Gesetz über die Gleichbehandlung von Mann und Frau auf dem Gebiete des burgerlichen Rechts (GleichberG) vom 18.6.1957 (BGBI. I, S. 609).

12 Die Divergenzen zurn auslandischen Recht sind kein Problem, de:n sich nur dic Bundesrepublik gegenuber sieht. Europaische Nachbarstaaten sind in ähnlicher Weise betrolfen, vgl. dazu Wengler (IPRax 1984, 177) zu einer niederlandischen Entscheidung bezuglich marokkanischer Moslems sowie einer englischen Entscheidung betreffend ene pakistanische Familie (IPRax 1985, 334).

13 Vgl. BGHZ 60, 68 = BGH FamRZ 1973, 138.

14 Das Abkommen tst am 17.9.1971 fur die Bundesrepublik in Kraft getreten (BGBl. 1971 II, S. 219).

is Spezielle Ordre-public-Normen enthalten Art. 16 MSA und Art. 8 III 2 des deutsch-iranischen Niederlassungsabkommens (NA). 
der lex patriae ausschaltet, wenn sie deutschen Rechts- und Werteverständnissen zu sehr widerspricht. ${ }^{16}$

Insbesonderc hat der BGH bei eincr Entscheidung ${ }^{17}$, in der es um die Regelung der clterlichen Sorge nach Scheidung eines in Deutschland lebenden iranischen Paares ging, befunden, daß das Grundrecht des Kindes auf Entfaltung seiner Persönlichkeit aus Artt. I I und 2 I GG schon dann verletzt sei, wenn das Sorgerecht bei Scheidung nicht demjenigen Elternteil zugesprochen werde, bei dem das Kind vermutlich die meiste Unterstützung für den Aufbau seiner Persönlichkeit erhalten könne. Überdies verstößt nach Ansicht des BGH eine Sorgerechtsentscheidung, die nicht das Wohl des Kindes zum entscheidenden Maßstab macht, sondern eine typisierende Regelung solange geltcn läßt, wie sie das Kindeswohl nicht gefährdet, gegen die staatliche Pflicht, Pflege und Erziehung des Kindes zu überwachen (Art. 6 II GG). Auf dic Frage, ob die iranische Vorschrift auch gegen den Gleichberechtigungsgrundsatz aus Art. 3 II GG verstoße, ging der Familiensenat nicht cin. Das tat cr dafür in ciner Entscheidung, in der es um die Personensorge für cinen Sohn iranischer Eltern ging, die seit 1986 in der Bundesrepublik lebten. ${ }^{18}$ Der Junge war nach der Scheidung der Mutter zugesprochen, die Vermögenssorge aber dem Vater vorbehalten worden. Die Mutter begehrte das alleinige Sorgerecht. Dicsmal prüften die Richter auch dic Verletzung von Art. 3 II GG, verneinten diese aber. Denn auch bei der Erörterung eines Verstoßcs gegen den Gleichberechtigungsgrundsatz sei nur zu prüfen, ob dic Anwendung des iranischen Rechts im konkreten Einzelfall den ordrc public verletze. Auch hier sei das Kindeswohl als Maßstab heranzuziehen. Stellt danach cine Entscheidung über die elterliche Sorge auf das Wohl des Kindes ab, "so ist der Elternteil, der ganz oder teilweise von der elterlichen Sorge ausgeschlossen ist, nicht in seinen Grundrechten verletzt. «' ${ }^{19}$ Ein Elternteil habe keinen verfassungsrechtlichen Anspruch auf Übertragung der vollen elterlichen Sorge.

Dicse beiden Beschlüsse machen deutlich, daß für den BGH bei der Sorgerechtsentscheidung das Kindeswohl ein wichtigerer Maßstab ist als die Verwirklichung des Gleichberechtigungsgrundsatzes. Sie belcgen, wie stark die Elternrechte nun dem Kindeswohl nachgeordnet werden. ${ }^{20}$ Somit haben diese zwei Entscheidungen des $\mathrm{BGH}$ die juristische Beurteilung eines Ordre-public-Verstoßes auf drei Ebenen verändert. Zum einen hat eine Akzentverschicbung zwischen den Grundrechten stattgefunden, indem nun deutlich das Kindesinteresse in den Vordergrund und das Elterninteresse in den Hintergrund tritt. Ferner wird das Kindeswohl als alles überragendes Richtmaß mit Verfassungsrang installiert. ${ }^{2 ~ S c h l i e ß l i c h ~ w i r d ~ d i e ~ V o r b e h a l t s-~}$ klausel auch auf Familien mit rein ausländischen Beteiligten erstreckt. ${ }^{22}$ Die frühere Relativierung der Grundrechte für Fälle mit Auslandsberührung ${ }^{23}$ ist damit weitgehend obsolct. Dem Respekt vor der fremden Rechtsordnung wird nun lediglich auf

16 Vgl. auch Palandt/Heldrich Art. 6 EGBGB Rn. 1; Staudinger/Blumenwitz. Art. 6 EGBGB nE Rn. is; Wolf, FamRZ $1993,874,875$.

$17 \mathrm{BGHZ} 120,29=\mathrm{BGH}$ FamRZ $1993,316$.

18 BGH FamRZ 1993,1053 .

19 BGH FamRZ 1993,1053 .

2o Die Rechte der Mutter in binationalen Fallen noch berucksichtıgend: OLG Karlsruhe (IPRax 1993, 97, 99), das einen Verstoß gegen Art. 3 II GG annummt, wenn die deutsche Mutter aufgrund der starren Regelung im algerischen Recht an der Sorgerechtsausubung ganz oder teilweise gehindert wird; ebenso das OLG Dusseldorf (NJW-RR 1994, 7, 8), welches die Rechte der Mutter wabren will, wenn sie wegen fehlender Religıonszugehorıgkeit zum Islam in Jordanien von der Sorge ausgeschlossen werden soll.

2 1 Eine Entwicklung, die sich auch schon in jungeren Entscheidungen der Instanzgerichte abzeichnete, vgl. nur OLG Stuttgart DAVorm 1986, 556, 559; AG Einbeck FamRZ 1991, 590, 592; OLG Saarbrucken FamRZ 1992, 848; OLG Karlsruhe IPRax 1993, 97, 99 f; fruher bereits einmal LG Hechingen IPRspr. 1966-67 Nr. 108; im Anscbluß an den BGH dann auch OLG Dusseldorf NJW-RR 1994, $7,8$.

22 Vgl. auch Fischer, RabelsZ 1993, 1, 12; Spickhoff, JZ 1993, 210.

23 So noch BGH FamRZ 1973, 138. 
der Ebene des Ersatzrechrs nach vorgelagcrter Ausschaltung der fremden Rechrsordnung Tribut gezollt. ${ }^{24}$ Weithin werden Sorgerechtsfälle mit auslandsbezogenem Sachverhalt jetzt nicht anders gchandhabr als reine Inlandsfälle. Über das Eingreifen der Korrektivklausel entscheidet in Sorgerechtsfragen eine Parallelbeurteilung des Sachverhaltes aus der Sicht des deurschen Rechts. ${ }^{25}$ Damit ist auch die Möglichkeit der Eltern, ihre Vorstellung von angemessener Erziehung durchzusetzen, stark eingeschränkt.

Diese Zentrierung auf die Kindesinteressen und die Gleichbehandlung deutscher und ausländischer Familien unter strengerer Handhabung des ordre public hat in der Literatur zu den beiden BGH-Beschlüssen überwiegend Beifall gefunden. ${ }^{26}$ Demgegenüber hat vor allem Rauscher davor gewarnt, mit dem Instrument des ordre public den Migrantenfamilien allzu rigoros deutsches Recht und deutsche Vorstellungen von bestmöglicher Berücksichtigung des Kindeswohls zu oktroyieren. Dahinter stecke die strenge und intolcrante Auffassung, die allein tragbare Lösung sei im deutschen Recht zu finden. Eine automatische Unwirksamkeit jeder Sorgerechtsentscheidung, die nicht strikt dem Kindeswohl nach der Idee des deutschen Rechts entspreche, sei daher abzulehnen. Denn das islamische Recht treffe seine Entscheidung in aller Regel keinesfalls »in blinder Bevorzugung des Vaters « ${ }^{27}$ Die. Ex-legeGcwaltverhältnisse des islamischen Rechts bevorzugten die Mutter in den frühen Jahren bei der Personensorge ebenso eindeutig wie dies die Statistiken bei der Sorgerechrszuteilung gemäß $\$ 167$ r BGB für deutsche Familien auswiesen. ${ }^{28}$

Damit ist zu Recht die Frage aufgeworfen, ob es sachgerecht ist, die ausländischen Familien bei der Beurteilung ciner Sorgerechtszuweisung genauso wie deutsche Familien zu behandeln. Denn diese vehemente Betonung der (Kindes-) Grundrcchte birgt die Gefahr eines "Grundrechtsoktrois «, ${ }^{29}$ der eine flexible Handhabung der Grundrechtsbindung bei auslandsbezogenen Sachverhalten zumindest erschwert. Diese Entwicklung übersieht, wic cs der BGH $197^{\text {jo }}$ richrig erkannt hat, zum einen, daß im internationalen Kindschaftsrecht bi- und mulrilaterale Verträge existieren, die ad absurdum geführt würden, könnte unter Verweis auf die deutschen Grundrechte der Einsatz fremden Rechts rundweg ausgeschlossen werden. Mit dem Beitritt zu den Staatsverträgen ist hingegen ein Stück weit der Verzicht auf die vollständige Durchsetzbarkeit der cigenen Rechtsstandards erklärt worden. ${ }^{31}$ Zum anderen ist bei der Suche nach einer Antwort auf diesc Fragen zunächst zu überlegen, ob das Sozialisationsprofil dcr zweiten Ausländergeneration eine solche Gleichbehandlung mit deutschen Minderjährigen rechtfertigt. Von der ersten Migrantengeneration ist dabei bekannt, daß sie heute noch weitgehend im ethnischen Verbund lebt und sich ihr Leben cmorional und geographisch um Landsleute zentriert, häufig um Verwandtc und Bekannte. Eine soziale Integration zwischen Deutschen und Ausländern der Elterngeneration hat faktisch kaum stattgcfunden. ${ }^{32}$

Nun ist es aber keineswegs so, daß die soziale Identität der zweiten Ausländergene-

2 . Dies ist dic neue Idee des BGH FamRZ 1993, 316, 318.

25 Staudinger/Coester $\$ 1671$ Rn. 222; Coester, IPRax $1991,236$.

26 Henrich, IPRax 1993, 81; Wolf, FamRZ 1993, 874; Spickholf, JZ 1993, 2 10; Berger LM Nr. 3 (zu Art. 6 EGBGB 1986$)$.

27 Rauscher, JR 1994, $184,188$.

28 Ullmann, FamRZ $1987,434,437$

29 Stern, Staatsreche $111 / 1 \$ 72 \mathrm{~V} 7$.

30 BGH FamRZ 1973,138 .

31 Rahm/Pactzold, Handbuch des Famuliengerichesverfahrens VIII, Rn. sis; aA Henrich, FamRZ :974, ros, 1 10; Spickhoff, JZ 1993, $210,212$.

32 Vgl. dazu insgesamt die Untersuchungen von Rosen/Stuwe, Auslandische Madehen in der Bundesrepublık ( 1985 ) und Stickelmann/Stuwe, Maßnahmeplan fur multıkulturelle Jugendarbeit in Frankfurt/Main (1992) 
ration, ${ }^{33}$ die zum überwiegenden Teil in der Bundesrepublik aufgewachsen ist, eindeutig durch die Kultur und die Sozialisationsinstanzen des Aufnahmestaates geprägt ist. Zwar setzt dies die Rechtsprechung in ihren neuen Entscheidungen stillschweigend voraus, vielmehr jedoch ist die Identität der jungen Migranten diffus und nicht mehr so eindeutig zuordnungsfähig wie bei den Eltern. Die nachwachsende Generation ist an vielen Punkten, die entscheidend ihre Sozialisation prägen, gespalten. Die Jugendlichen versuchen stets, die Anforderungen, welche von der Herkunftskultur durch ihre Eltern an sie herangetragen werden, mit den Erfordernissen der Residenzkultur, die durch Freunde, Schule und Beruf vermittelt werden, in Einklang zu bringen. Dieser kulturelle und emotionale Spagat gelingt nicht immer. In Zweifelsfällen entscheiden sich die jungen Ausländer häufig für die Lösung, die ihnen ihre Herkunftskultur nahelegt: Sie haben im Freizeitbereich vor allem Kontakt zur eigenen Ethnie und bleiben auch bei der Wahl des Lebenspartners eher unter sich. Insgesamt dominiert die Konzentration auf Kontaktpersonen und Institutionen der ethnischen Community. Die bei der Elterngeneration festgestellte Isolierung im deutschen Unfeld ist nur bci einem Tejl der zweiten Generation aufgebrochen worden. Deutsche Jugendliche und solche aus Migrantenfamilien leben mehr neben- als miteinander. So sind auch die Beziehungsstrukturen durch ethnische Segmentierung geprägt; sie sind relativ abgeschlossen gegenüber der Mehrheitsgesellschaft. Auch die nationale Selbsteinstufung ist bei der zweiten Generation deutlich. Sie fühlt sich im Prinzip ihrer ethnischen Gruppe zugehörig. Diese Tendenz scheint sogar im Moment stärker zu werden. Jugendliche der zweiten und dritten Generation besinnen sich zunehmend ihrer ethnischen Wurzeln, sehen ihre Zukunft jedoch in Deutschland. ${ }^{34}$ Auffällig dabei ist, daß ein Fünftel der Ausländer der zweiten Generation in einer neueren Untersuchung häufig das Gefühl von Heimatlosigkeit äußert. ${ }^{35}$ Darüber hinaus erlebt die zweite Generation, trotz oft gleicher Qualifikation und guter Sprachkompetenzen, erhebliche Benachteiligungen auf dem schulischen und beruflichen Sektor.

Aus alledem folgt: Die Bedingungen und Chancen der zweiten Generation haben sich im Vergleich zu den Eltern nicht automatisch und entscheidend verbessert. Die nachgewachsenc Generation lebt zwischen den Kulturen. In vielen Lebenssituationen bleibt die Herkunftskultur handlungsleitend. Aufgrund dieser Resultate ist nicht von einer gelungenen Integration der zweiten Generation in die deutsche Gesellschaft zu sprechen. Die jungen Menschen ziehen sich vielmehr weithin in ihre ethnische Gemeinschaft zurück. Diese Community ist für die zweite Generation der Raum, in dem sie die Traditionen ihrer Eltern ernstgenommen sieht, der Platz für herkunftsbezogene Bildung und (Sekundär-) Sozialisation, ein soziales Auffangnetz bei mißlungener Integration und ein Forum der Beratung und der Verbindung mit Gleichaltrigen.

Außerdem ist zu beachten, daß dieser Befund nicht ausschließlich eine Reaktion auf existierende oder vermutete Diskriminierung durch die Deutschen ist. Vielmehr wird er auch zu einem guten Teil getragen von einer nicht immer ausgebildeten Integrationsbereitschaft auf Seiten der Migranten. Dieser mangelnde Assimilierungswunsch verbindet sich mit einem Bedürfnis nach Anerkennung in einem kollektiven Sinne. Es ist der Wunsch nach Achtung vor den Handlungsformen, Eigenarten, Praktiken und Besonderheiten der Weltanschauung, die für Angehörige ethnischer Gruppen große Bedeutung haben. ${ }^{36}$ Die Forderung nach kollektiver Anerkennung

33 Mitclerweile wächst bereits die dritte Migrantengeneration heran.

$34 \mathrm{Vg}$. dazu instruktiv Süddeutsche Zettung vom 31.7.1995 (*Einmal Bosporus, aber bicte retour $)$.

3s Seifcrt, KZfSS $1992,677,683$

36 Habermas (Fn. 3), S. 150 . 


\section{XRITISCHE JUSTIZ}

Herausgcber und Kedaktion: Thomas Blanke, Petcr Derleder, Raıner Erd (verantw.), Gunter Frankenberg, Monika Frommel, Rolf Knicper. Ulrich Muckenberger, Joachın Perels, Sibylle Razsch, Jürgen Scifer, Ulrich Stascheit, Margaretha Sudhol, Raner Woll.

Unter Mularben von: Hartmut Baumer. Uwe Berlit, Hans-Timst Botteher, Alexander v. Brunncek, Marlies Durkop, Klaus Groth, Heinfich Hannover, Dicter Hart, Ratner Keller, Stephan Leibiried, Wolf-Dietcr Narr, Ulrsch K. PreuB, Chrisloph U.Schminck-Gustavus, Dıeter Sterzel, Uwe Wesel, Gerd Winter.

Redakuonelle Zuscinfien and Besprechungsexemplare bituc an: Joachım Perels, Kanestr. 4, 3062 s Hannover, Tel.: os 11/ss 6644 ; Thomas Blanke, Werbachser. 32, 26121 Oldenburg, Tel.: $0441 / 84648$. Die Redakuon bitce die Lesennnen und Lescr um Mitarbcit an der Krizischen Justız, kann aber fur unverlangt eıngesandic Manuskripte keıne Haftung vibemehmen. Beitrage sollen die Lange von as maschinenschnftichen Seiten (einseiug, ziverzeilig, 60 Anschlage pro Zeile) nicht uberschreiten. Dic Redakuson betsc, Manuskripte in dreifacher Ausierugung zu ubersenden. Lieraurhinwesse wie in der Knuschen Justuz ublich. Unverlangt eingesandie Besprechungsexemplare konnen niche zuruckgesande werden.

Namentush gezerdonete Bestruge geben niclue in jedem Fall die Mcinung der Merausgeber/Redakuon oder des Verlagcs wieder.

Bezugsbedengungen: De Zeitschrift erscheint vierreljahrhch. Abonnemene jihrlich 48,- DM zuzuglich Zustellgebuhr; fur Seudenten (jahrlıche Vorlage einer Studienbescheinigung erforderlich) jahthels 38,- DM zuzugglich Zusecllgebuhr; Einzelheft 14,50 IJM Abbestellungen mussen spatestens drei Monate vor Ende des Xalenderjahres schriftlich erfolgen. Die zur Abwieklung von Abonnements erforderischen Daten werden nach den Beseummungen des Bundesdaicnschueageserzes verwalter.

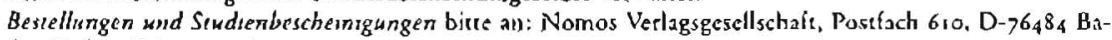
den-Baden, Tel. $07221 / 2104-0$.


Verwerung, die niche ausdrucklich vom Urheberrechesgesetz zugelassen ist, bedarf der vorherigen Zustimmung des Verlags. Das gilr insbesondere fur Verviclfalligungen, Bearbeitungen, Ubcrscizungen, Mukroverfilmungen und Einspeicherung und Verarberrung un elektronischen Systcmen.

Verlag: Nomos Verlagsgisellschale mbH \& Co. KC, Baden-Baden.

Salz: Buch und Offsetdruckerei Wagner GmbH, Nordlingen.

Druck: Nomos Verlapsgesellschare mbH \& Co. KG, Baden-Baden, Dructehnus Sinxhemm 


\section{Winfried Hassemer/Klaus Peter Möller \\ 25 Jahre Datenschutz}

Bestandsaufnahme und Perspektiven

Ein Vierteljahrhundert Datenschutz in Hessen: Das erste Hessische Datenschutzgesetz von 1970 war nicht nur das erste Gesetz seiner Art in der Bundesrepublik, sondern hatte eine weltweite Pionierfunktion. Die in diesen Band aufgenommenen Beiträge beleuchten das Gesetz unter dem Blickwinkel eines Vor- und Leitbilds zur theoretischen und praktischen Entwicklung eines Menschenrechts auf Schutz der persönlichen Daten.

Der rasante technische Fortschritt auf dem Gebiet der neuen Technologien - Multimedia, weltweite Kommunikation z.B. auf dem INTERNET oder die stürmische Verbreitung der Datenverarbeitung in privater Hand mit dem Personalcomputer - zwingt die Datenschutzbeauftragten zur Entwicklung neuer Konzeptionen. Das 1995 veranstaltete 4. Forum Datenschutz, dessen Vorträge und Diskussionen dieser Sammelband wiedergibt, hat daher auch eine Fülle von Ideen entwickelt, die sich - auf der Basis überkommener Traditionen - zu einem neuen Konzept des Datenschutzes zusammenfügen.

1996, 80 S., brosch., 28,-DM, 207,50 öS, 25,50 sFr, ISBN 3-7890-4123-8

(Forum Datenschutz, Bd. 4)

\section{NOMOS VERLAGSGESELLSCHAFT} Postfach 610 • 76484 Baden-Baden

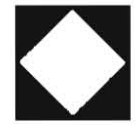


verfolgt dabei vorrangig das Ziel, die Unverletzlichkeit der Lebensformen und Traditionen, in denen sich die Angehörigen benachteiligter Gruppen wiederfinden, zu schützen. So zeigt sich, daß ein Bedürfnis bei den Migranten existiert, sich nach heimatlichem Muster zu organisieren und dies auch rechtlich durchzusetzen. Dem wird aber - wie hier aufgezeigt - eine Rechtsprechung aufgesetzt, die dies nicht berücksichtigt und zudem die vorhandene soziale Diskrepanz zwischen dcutschen und ausländischen Jugendlichen tendenziell einebnet.

Dieser Widerspruch macht es unabweisbar, über ein gesellschaftstheoretisches Konzept nachzudenken, das den kulturellen Minoritäten die Möglichkeit eröffnet, ihr Zusammenleben und dessen rechtliche Regulierung bis zu einem gewissen Grad autonom zu gestalten. Juristisch geht es dabei um die Frage, unter welchen Aspekten ein Zusammenleben von einheimischer Bevölkerung und zugewanderten Minderheiten gestaltet werden soll und welche Rolle in diesem Zusammenhang dem Familienrecht zukommt. Ist es möglich, Migrantenfamilien bei der Regelung ihrer ElternKind-Konflikte zumindest teilweise von der Anwendung deutschen Rechts auszunehmen und im Einzelfall das Heimatrecht stärker zu beachten? Ist also ein Konzept denkbar, das es den Familien ethnischer Minderheiten erlaubt, auch in der Bundesrepublik nach ihrem Heimatrecht zu leben? Damit ist auch die Frage nach der Zulassung von Sonder- und Gruppenrechten für Minoritäten gestellt.

\section{Politik der Differenz - Modell für die Zukunft?}

Eine solche Form von kollektiven Rechten für Minderheiten ist aber im Konzept deutscher Staatlichkeit nicht von vornherein angelegt. Denn die Bundesrepublik ist ihrem überkommenen Selbstverständnis nach ein Nationalstaat, der auf der politischen Ideologie des ethnischen Nationalismus bcruht. ${ }^{37}$ Dieses Staatsverständnis prägt auch den Umgang mit Minoritäten. So ergibt es sich, daß diese Form des Nationalismus, der ethnische und kulturelle Homogenität anstrebt, andere ethnische Gruppen als Störfaktor empfindet, die im besten Fall geduldet werden können. Sie bedrohen gleichsam die nationale Einheit. ${ }^{38} \mathrm{Um}$ die eigene Identiät und den sozialen Frieden zu bewahren, muß die fremde Kultur bekämpft oder negiert werden. ${ }^{39}$ Deshalb ist im ethnischen Nationalismus eine starke "Tendenz zur Assimilierungspolitik " $^{\circ}$ angelegt, durch die der Staat versucht, die nationale Einheit herzustellen und ethnische Minderheiten als selbständige Gruppen aufzulösen."' Der Faschismus trieb diese Ideologie auf die Spitze und suchte, die kulturellen Minoritäten zu vernichten.

Vor allem dem Freiburger Politologen Dieter Oberndörfer ist es gelungen, die Relikte des völkisch-ethnischen Nationalismus im Grundgesetz herauszuarbeiten. ${ }^{42}$ Die Bundesrepublik wurde als Republik für "Deutsche" gegründet. In Widerspruch zu seinem eigenen republikanischen Wertefundament bezog sich das Grundgesetz

\footnotetext{
37 Heckmann, Ethnische Minderheiten, Volk und Nation (1992), S. 4, 211.

38 Oberndorfer, Die offene Republik (1991), S. 35 .

39 Schulte (Fn. 7), S. 7.

40 Heckmann (Fn. 37), S. 212

41 Das Deutsche Reich von 1871 wollte zum Beispiel die Ruhrgebietspolen und die danische Minderhert in Schleswig-Holstein germanisieren. Die Weinarer Republik erkannte zwar echnische Minderheitenrechte in der Verfassung an, doch es gab kaum Erlasse oder Verordnungen zum Minderheitenschutz etwa in Schulwesen oder der Verwaltung, vgl. Heckmann (in. 37), S. 213.

42 Erstmals Oberndorfer, ZAR 1989, 3, 8 ff; spater dann in Oberndörfer (Fn. 38), S. 59 ff.
} 
des neuen Staates auf ein "völkisch-nationales Substrat«, ${ }^{43}$ Daher war die neu geschaffene Republik nur eine vorläufige, behelfsmäßige staatliche Notordnung für dic in Freiheit lebenden Deutschen. Und mit dem Wiedervercinigungspostulat bewegte sich die Verfassung der neuen Republik in der Tradition des ethnischen Nationalismus. ${ }^{44} \mathrm{Im}$ Gegensatz dazu stehen die für alle gültigen, in der Tradition der Menschenrechte stehenden Grundrechte, dic in Art. I GG auf die Würde des Menschen bezogen werden. Folgerichtig sind daher in Artt. 2, 3 und 4 der Verfassung die Grundrechte der Frciheit der Person, der Gleichheit vor dem Gesetz, der Glaubensund Meinungsfreihcit "jedem « und "allen "Menschen gewährt. Demgegenüber ist in den Bestimmungen zur Versammlungs-, Vereinigungs-, und Berufsfreiheit sowie der Freizügigkcit nur von Rechten »für alle Deutschen « die Rede. Wirtschaftlich und politisch grundlegende Rechte wcrden somit allcin den Dcutschen vorbehalten. Gerade hier wird das ethnische Relikt im Republikverständnis des Grundgesetzcs deutlich. ${ }^{45}$ Diese in der Verfassung angelegte Spannung zwischen weltoffenen, republikanischen und ethnischen Orientierungen zeigt sich auch an der renitenten Negation der Einwanderungssituation in der Bundesrepublik durch die politisch Verantwortlichen. Sie wiederholen gebetsmühlenhaft die Formel: „Die Bundesrepublik ist kein Einwanderungsland. ${ }^{4}{ }^{6}$ Zudem belegen Art. I 16 GG (mit der Staatsbürgerschaft der Vertriebenen und Volksdeutschen) sowie dic Weigerung, die doppelte Staatsbürgerschaft zuzulassen und die Einbürgerung grundlegend zu vereinfachen, daß sich die Bundesrepublik noch immer als ethnische Abstammungs- und Kulturgemeinschaft versteht. ${ }^{+7}$

Dieses Verständnis prägt natürlich auch die Bedingungen, die an das Zusammenleben mit dauerhaft in der Bundesrepublik niedergelassenen Ausländern gestellt werden. Es wird ihnen eine Integrationsvorleistung abgefordert, die zum Ziel hat, daß sie zumindest partiell ihre Herkunftskultur aufgeben. Das heißt, die Einwanderer sollen Orientierungen und Verhaltensweisen wie Ehrgeiz und Organisationstalent, Ordnungssinn, Gemeinnützigkeit und die deutsche Einstellung zur Arbeit übernehmen, die zu den kulturellen Standards der Bundesrepublik gehören, also sich so etwas wie "national wesentliche Eigenschaften $\alpha^{4^{8}}$ aneignen. Nur im pcrsonalen Bereich soll die Heimatkultur respektiert und geschürtzt werden. ${ }^{49}$ Mit diesen Voraussetzungen für eine Integration wird - jedenfalls implizit - die Höherwertigkeit der als "deutsch " bezeichneten soziokulturellen Orientierungsmuster unterstellt. ${ }^{50}$ Abgelehnt wird damit gleichzeitig eine Idee der multikulturellen Gesellschaft, die die Gleichberechtigung aller Herkunftskulturen von Eingewanderten mit der »überkommenen deut-

43 Oberndorfer (Fn. 38), S. 60.

44 Obcrndorfer, Vom Nationalstaat zur offenen Republık - Zu den Voraussetzungen der politischen Integration von Einwanderung, in: Aus Polıtık und Zeitgeschichte, Beılage zur Wochenzeltschrift "Das Parlamenta, Februar 1992 B 9, S. 24.

45 Oberndorfer (Fn. 38), S.61.

46 Vgt. nur das als "Regierungsprogramm - bezeichnete Wahlprogramm der CDU/CSU fur die Bundestagswahl vom 16.10. 1994, in: Die Zett vom 25. 8.1994: "Deutschland ist keın Einwanderungsland. Eine unbcgrenzte und nicht steuerbare Zuwanderung nach Deutschland lehnen wir entschieden ab. Ein Einwanderungsgesetz wird es mir uns nicht geben-. Siehe auch die Begrundung des Gesetzentwurfs der Bundesregierung zum neuen AuslG in BT-Drs. 11/6321, S. 41 ("Dic geseczliche Regelung des allgemeinen Auslanderrechts gehe von der weiterhin grundsatzlıchen Entscheidung aus, daß die Bundesrepublik Deutschland nicht unbegrenzt allen zuwanderungswilligen Auslandern einen dauernden Aufenthalt ermoglich kann.\&)

47 Zur lllustrterung mägen nur die Worte des Vorsitzenden der CDU/CSU-Bundestagsfraktion, Wolfgang Schauble, dienen: "Wir - che alten Staten des alten Europa - sind klassısche Nationalstaaten. Wir schopfen unsere Identirat nicht au detn Bekeinntnis zu ciner Idee, sondern aus der Zugehorigkeit zu einem bestummten Volk. \& (zitiert nach Die Zeit vorn 2. 2. 1996)

48 Quaritsch (Fn. 4) nenut diese Eigenschafen *Industrictugenden *.

49 Schitter, in: Der Spregel. Hefe $40 / 1991$, S. 55

so Schulte (Fn. 7), S.8. 
schen Kultur auf dem deutschen Territorium "' bedeuten würdc. Abgelehnt werden kulturelle Enklaven, in denen die Migranten nicht nur in dem als privat verstandenen Bereich nach ihren Traditionen, Riten, sondern eben auch nach der Rechtsordnung ihrer Herkunftskultur leben können. ${ }^{52}$ Frcmde, Ausländer, ethnische Minderhciten können danach zu Bürgern zweiter Klasse gestempelt werden. ${ }^{33}$

Ein verändertes Umgehen, das dem Respekt vor cthnischen Minderheiten und der neuen Globalsituation Rechnung trägt, wäre in einem Staatskonzept der offenen Republiks denkbar, dem ein politisch-republikanisches Nationkonzept zugrunde liegt. Die offene Republik ist der extreme Gegentyp zum erhnischen Nationalstaat. Sie hat cin weltbürgerliches Wertcfundament'ss und leitet die Rechte, die sie ihren Bürgern gewährt, aus allgemeingültigen, für die. Menschen aller Völker geltenden "Menschenrechten " her. Daher entscheiden auch Gemeinsamkeiten von Wertvorstellungen, Institutionen und politischen Überzeugungen, jedoch nicht eine gemeinsame Abstammung über die Zugehörigkcit zur Nation. ${ }^{56}$ Bürger der republikanischen Nation können grundsätzlich alle Menschen werden, die dies wünschen und sich zur republikanischen Verfassung bekennen; die freiwillige Beteiligung der Einzelpersonen bildet die Nation. 77 In der offenen Republik bedarf daher das Recht auf Einwanderung und Einbürgerung keiner eigenen verfassungsrechtlichen Begründung..$^{8}$ Der derart definierte republikanische Verfassungsstaat stellt zunächst nur einen Idealty pus dar, der ein verändertes Umgehen mit den ethnischen Minderheiten versinnbildlicht. Dies bedeutet jedoch nicht, daß er sich für unkontrollierte und unbegrenzte Einwanderung öffnen muß. Einwanderung findet daher unter der Prämisse statt, daß sie die Überlebensfähigkeit der politischen, rechtlichen und sozialen Ordnung der offenen Republik nicht gefährdet. $\$ 9$

Der republikanische Verfassungsstaat ist demnach idealiter eine politische Gerneinschaft für Menschen unterschiedlicher ethnischer Herkunft und kultureller Überlieferungen. Er ist somit nicht nur de facto, sondern auch von Rechts wegen multikulturell. Zwar finden sich in dem einen oder anderen Staat Elemente der so definierten politischen Nation, ${ }^{60}$ doch ist sie und die mit ihr verbundene "republikanische Weltkonföderation ${ }^{61}$ bisher weitgehend Utopie geblieben. Doch das stetige Zusammenwachsen der Welt, bedingt auch durch die wcltweiten Bevölkerungsverschiebungen, macht das Konzept als konkrete Gestaltungsaufgabe der Politik fast notwendig. Dem vermag auch letztlich die Renaissance des ethnischen Nationalismus in Teilen der Dritten Welt und Osteuropas nichts entgegenzusetzen. Die offene Republik ist die der Zukunft zugewandte Staatsform. Der ethnische Nationalstaat

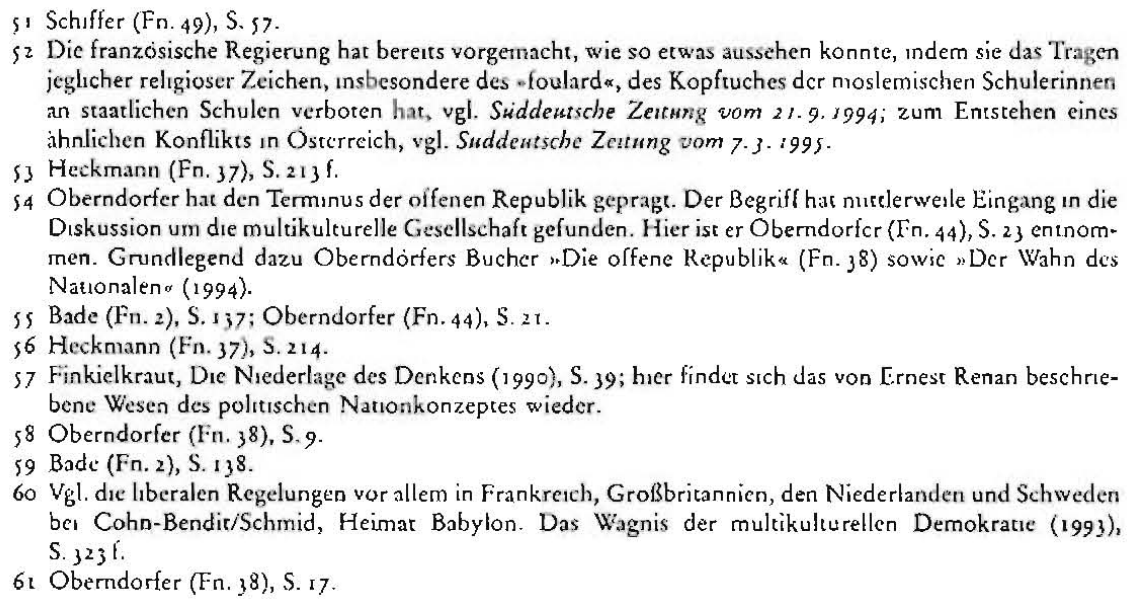


hingegen ist rückwärtsgewandt und nährt sich aus atavistischen Vorstcllungen des 19. Jahrhunderts, von einer Aufsplitterung der Menschheit in sich voneinander abschottende stammesartige Nationalstaaten.

Es fragt sich aber, in welcher Weise diese ethnische Pluralität in der offenen Republik zu organisieren ist. Dies ist in der Weise denkbar, daß der Staat die Beibehaltung und Förderung ethnischer Vielfalt erlaubr. ${ }^{62}$ Eine solche Politik der Differenz interpretiert Rechtsgleichheit nicht expansiv. ${ }^{6}{ }_{3}$ Der Staat regt die ethnischen Gruppen dazu an, sich als solche zu organisieren und ihr Gewicht in Politik und Staat geltend zu machen sowie einen formell-rechtlichen Minderheitenstatus zu erreichen. ${ }^{64} \mathrm{Kultu}-$ relle und ethnische Differenzen werden so institutionalisiert und intensiviert. ${ }^{65}$ Die dieser Politik zugrunde liegende Variante eines politischen Liberalismus hat vor allem der kanadische Sozialphilosoph Charles Taylor entworfen. ${ }^{66}$ Danach ist der Staat (in multikulturellen Gesellschaften) nicht streng neutral, sondern parteilich und setzt sich für den Fortbestand und das Gedeihen einer bestimmten Nation, Kultur oder Religion oder einer begrenzten Anzahl von Nationen, Kulturen oder Religionen ein ${ }^{67}$ Dabei sichert er allerdings die Grundrechte jener Bürger, die sich in anderer Weise oder gar nicht engagieren wollen. Er schützt »elementare Freiheiten, die niemals eingeschränkt werden dürfen «. ${ }^{68}$ Taylor nennt exemplarisch das Recht auf Leben, auf Freiheit, fairen Prozeß sowie die Rede- und Religionsausübungsfreiheit. ${ }^{69}$ Von diesen klassisch-liberalen und elementaren Grundrechten seien aber Vorund Sonderrechte zu unterscheiden, »die zwar wichtig sind, aber aus politischen Gründen, allerdings nur aus sehr triftigen, widerrufen oder beschnitten werden können. " $^{70}$

Liberalismus der Taylorschen Prägung wägt im Ergebnis bestimmte Formen von Gleichbehandlung gegen kulturelle Differenzrechte ab. ${ }^{71}$ Taylor verneint somit eine "ethische Neutralität « der Rechtsordnung. ${ }^{72}$ Der Staat darf bestimmte Konzeptio-

62 Offizielle Doktrın in Einwanderungsgesellschaften wie denen der USA und Kanada, aber auch grobe Leitlinie der Polıtk in der Bundesrepublik ist allerdings ein auf Individualrechte zugeschnittenes Liberalismus-Modell. Theoretisches Fundament solcher Politik sst ein polituscher Liberalismus, der sıch so nachdrücklich wie moglich fur die Rechce des cinzelnen einsetzt, und gewissermaßen als logische Folge hiervon auch für einen streng neutralen Staat, einen Staat also ohne eigene religıose und kulturelle Projekte oder kollektive Ziele. Dieser Liberalismus sorgt furr die Gleıchhett der Rechte aller Burger und sichert nur, daß jedes Individuum seine Konzeption des "Guten* verwirklichen kann. In der Gegenwart ist dieses liberale Modell vor allcm von dem US-amerikanischen Sozıalphilosophen und Staatstheoretiker John Rawls entwickelt worden, vgl. dazu insgesamt Eine Theorie der Gerechtigkeit (r97s). Ein liberaler Rechisstaat muß danach im Hinblick auf die Idee des guten Lebens eines jeden Bürgers neutral bleiben. Er hat lediglich zu gewahrleisten, daß die Burger, gleichgultig, welche Anschauungen sie haben, fair miteinander umgehen. Daruber hinaus muß der Staat alle glerchbehandeln und gleiche Ausgangsbedingungen fur alle schaffen. Sonder- und Gruppenrechte aufgrund der Zugehorigkeit zu einer bestimmten Erhnie haben hicr keinen Platz. Aus der Sicht dieses Liberalismus gıbt es kerne privilegierte Majoritat und kenne Minoritat nit Sonderstatus.

63 Von einem ethnischen Korporatismus sprıcht etwa Walzer, Pluralism in Political Perspective, in: Thernstrom, Stephan: (Hrsg.): The Politics of Ethnicity (1982), S. 24; vgl. auch Brumlik/Leggewic, Konturen der Einwanderungsgesellschaft: Nationale Identitat, Multikulturalismus und ${ }$ Civil Society $\alpha$, in: Bade, Klaus J.: Deutsche im Ausland - Fremde in Deurschland (1993), S. $43^{8 \mathrm{ff}}$.

64 Walzer (Fn. 63), S. 20 ("where ethnic groups no longer organize themselves like voluntary associations but have instead some poliucal standing and some legal rights $\%$ ).

65 Walzer (Fn. 63), S. 24 ("The effect of auconomy would be to intensify and instututionalize cultural differences).

66 Ausfuhrlich in semem Essay "Multıkulturalismus und die Politik der Anerkennunga von 1993, S. ${ }_{13}$ ff., hier vor allem $S_{.43-56 .}$

${ }_{67}$ So auch Walzer (Fn. 63), S. 19 ("the state should defend collective as well as individual rights").

68 Taylor (Fn. 66), S. 53 .

69 Taylor (Fn. 66), S. 53 .

70 Taylor (Fn. 66), S. 53 .

7 T Taylor (Fn. 66), S. 56.

72 Gerade für diese ethische Veutralitat macht sıch aber Raw's (Fn. 62, vor allem S. 40ff.) in seinem L.beralismusmodell stark. II.thisch bezieht sich hier auf alle Fragen, die sich mu der Konzeption des guten Lebens befassen, vgl. Habermas (Fn. 3is. S. igi, 164 f. 
ncn des guten Lebcns fördern und ist nicht »differcnz-blind ". ${ }^{73}$ Insofern beinhaltet diese Strategie "grundrechtseinschränkende Statusgarantien ", ${ }^{74}$ welche das Überleben bedrohter kultureller Lebensformen erlauben. Danach kann ein Katalog von Grundrechten in einem bestimmten kulturellen Zusammenhang anders interpretiert werden als in einem anderen und so dazu dienen, verschiedene kollektive Ziele zu realisieren. ${ }^{75}$ In der Ziellinie solcher Politik, die Walzer eine wautonomistische Strategic ${ }^{76}$ genannt hat, liegen Gruppen- und Sonderrechte für Minoritäten. Materielle und ideelle Leistungen des Staates werden monopolistisch an die (dauerhafte) Zugehörigkeit zu einer Minderheit geknüpft, wobei überkommene ethnische Gruppenstrukturen bestätigt werden. ${ }^{77}$ Ethnizität wird also zum relevanten Merkmal der Leistungsberechtigung. Das Modell von Taylor strebt dabei eine Gesellschaft an, in der auch langfristig ethnische und kollektive Differenzen erhalten bleiben. Hinter der plakativen Formel Living with diversity steckt dic Idee eines dauerhaften harmonischen Miteinanders von ethnischen Kollektiven, Religionen, Weltanschauungen und Lebensweisen $;^{78}$ eine Vorstellung, die weniger durch den "melting-pot " als mehr - um im Bild zu bleiben - durch die "salad-bowl « symbolisiert wird. ${ }^{79}$ Auf der juristischen Ebene bedeutet dies, daß jeder einzelnen dieser Gruppen im Sinne Tay lors als "Gesellschaft mit besonderem Charakter "eine Form von Sonder- und Gruppenrechten unterhalb der nicht einschränkbaren Grundrechte zuerkannt werden könnte. So würde ihnen im Ergebnis die kollektive Anerkennung zuteil, die ihnen im anderen Liberalismusmodell versagt bleiben muß.

Die Politik der Differenz macht es dabei unabdingbar, in einer Gesellschaft mit starken multikulturellen Zügen diejenigen Werte herauszukristallisieren, die als solche unrelativierbar und daher für alle beteiligten ethnischen Gruppen verbindlich sind. Wie aber kann eine Gesellschaft als Konglomerat von ungleichartigen Gruppen politisch stabil bleiben und sich kulturell entwickeln ${ }^{20}$ Die Lösung muß in einer Art Konsens zur Organisation des Gemeinwesens gefunden werden. Die multiethnische Gesellschaft setzt somit die Existenz einer gewissen kulturellen Basis verinnerlichter Werte voraus. ${ }^{81}$ Diese sind als solche »überethnisch ${ }^{82}$ und daher für alle Gruppen akzeptabel und verpflichtend. Normen dieser Art übersteigen und ergänzen die kollektiven Werte jeder Gruppe und sind identisch mit den von Taylor beschriebenen »elementaren Freiheiten ", die nicht einschränkbar sind. Damit ist aber die Frage danach gestellt, wer diese Werte setzt; wer also bestimmt, was elementare Freihciten in der offenen Republik sind?

Die Antwort kann nur darin gefunden werden, daß unabhängig davon, wie bestimmte Ideale der Migranten in der Herkunftskultur zu bewerten sind, die aufnehmende Gesellschaft das Recht hat, ihre elementaren Freiheiten und Kernwerte zum Maßstab zu machen. Das muß allein schon aus dem Gedanken folgen, daß die Migranten sich in der Residenzgesellschaft aufhalten und dieser insofern ein Wertsetzungsprimat zukommt. Da sich die offene Republik aber durch ein weltbürgerlich

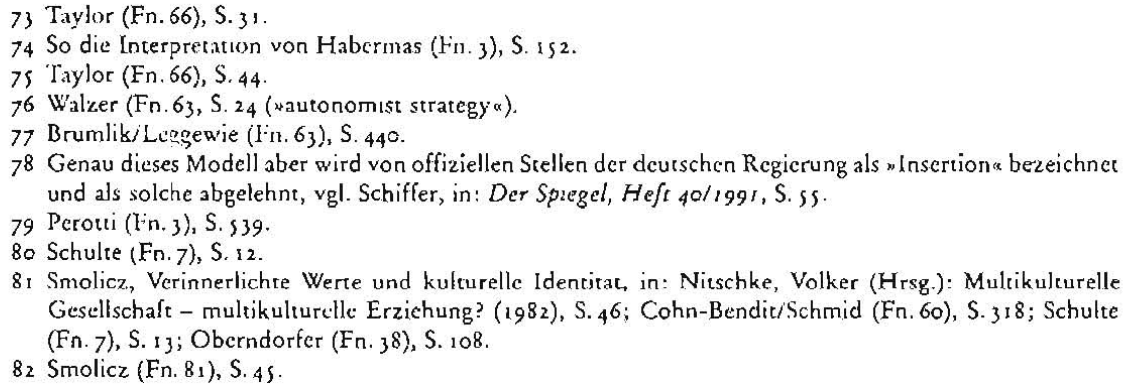


normatives Wertefundament auszeichnet, sind diesc Werte Ausfluß eines allgemeingültigen Grundbestands an Toleranz und daraus erwachsender Normen. Diese sind gleichzusetzen mit Menschen- und Bürgerrechten, die für kein Mitglied der offenen Republik eingeschränkt werden dürfen und daher universelle Geltung beanspruchen. ${ }^{8}$ Diese Grundnormen, die in liberalen Gesellschaften als konstitutiv begriffen werden müssen und Ausdruck in einer weltbürgerlichen Verfassung und Rechtsordnung finden, stellen somit zugleich die Grenze für die Frciheiten der an der multikulturellen Gesellschaft partizipierenden ethnischen Gruppen dar. ${ }^{84}$

Daher kann es im republikanischen Verfassungsstaat auch keinen unkritischen Kulturrelativismus geben, der sich jeglicher Bewertung bestimmter Elemente fremder Kulturen enthält. ${ }^{8}{ }^{8}$ Kulturelle Freiheit hat ihre Grenze dort, wo allgemeinverbindliche Menschen- und Bürgerrechte in Gefahr sind. Würde letztlich einem absoluten Kulturrelativismus das Wort geredet und die Kulturen so als umfassende Totalitäten begriffen, bedeutete dies, auf das gleiche Instrumentarium zurückzugreifen wie die Anhänger des ethnischen Nationalstaatcs. Das Wcltbild wäre dasselbe, nur die Glaubensbekennenisse gingen auscinander. ${ }^{86}$

Grundsäızlich muß in der Politik der Differenz der unterschiedslos geltende Katalog von Rechten anders ausgeprägt scin als in dem Modell eines streng neutralen Staates, damit so das Fortbcstehen ethnischer und kultureller Kollektive, verbunden mit der Wahrung und Entwicklung ihrer Identitäten in einem rechtlichen und sozialen Rahmen, möglich ist.

\section{Umsetzung auf die familienrechtlichen Konflikte}

Setzt man nun diese theoretischen Erkenntnisse auf die Eltern-Kind-Konflikte der hier beschriebcnen Art um, so bietct sich eine aufeinander abgestimmte, zweistufige Lösung an; zunächst ist dic Frage der anwendbaren Rechtsordnung zu klären, und zum zweiten ist gegebenenfalls die Frage des Einsatzes des ordrc public zu erörtern. So ist auf der ersten Stufe zu prüfen, ob auf der Ebene des anwendbaren Rechts die lex patriae oder die lex fori durchgrcift. Im Zentrum stcht dabei das Haager Minderjährigenschutzabkommen (MSA) ${ }^{87}$ und hier die konfliktträchtige Norm des Art. $3{ }^{88}$ die bei Existenz eines gesetzlichen Gewaltverhältnisses ${ }^{89}$ auch für ein in Deutschland

83 Lepenies (Fn. 3), S. 28; Taylor (Fn. 66), S. 53.

84 Bade (Fn. 2), S. 14 ; d dabei mogen sich unter diesen Grundwerten emige befinden, die in anderen Kulturen, deren Brauche wcit in die Geschichte zuruckreichen, nicht existieren, so etwa gewisse Grundrechte der Person. Zu denken ware hier an die Rechte-Ungleichheit von Frau und Mann im Islam. Vgl. dazu auch Finkielkraut (Fn. 57), S. 114 ; Tibi (Fn. 10), S. $178 \mathrm{ff}$.

8 s Schulte (Fn. 7), S. 10.

86 Finkielkraut (Fn. 57), S. 98; Schulte (Fn. 7), S. 9; in diesem Sinne auch Tibi (Fn. 10), S. 167, 173; Apel, Andersseın, ein Menschenrecht? Uber die Vereınbarkeit universaler Normen mit kultureller und ethnischer Viclfalt, in: Hoffmann/Kramer (Hrsg.): Anderssein, ein Menschenrecht (1995), S. 10.

87 Das MSA (vgl. auch Fn. 14) verdrangt als lex specialıs gemaß Art. 3 II I EGBGB die Regelungen des deutschen Internationalen Kindschaftsrechts fur seinen Anwendungsbererch. Fur emen Ruckgriff auf dic Normen des EGBGB verbleibt fur die hier zu behandelnden Konflikte fast kein Spiclraum mehr. Staudinger/Kropholler, Vorbem. i zu Art. ${ }_{9}$ EGBGB; Lorenz, IPRax 1992, 305, 30 s f. Raum bleıbt aber fur die Anwendung der Artt. 21, 22, 23 EGBGB (Legitimation und Adoption); vgl. ausgiebig zur Entstehungsgeschichte des MSA Allinger, Das Haager Minderjahngenschutzabkommen, Probleme, Tendenzen und Perspektiven (1988), S. 22 ff; Staudinger/Kropholler, Vorbem. 16 zu Art. 19 EGBGB.

88 Art. 3 MSA schreibe vor: Eın Gewaltverhaltnıs, das nach dem innerstaatlıchen Recht des Staates, dem der Minderjahrige angehort, kraft Gesetzes besteht, ist in allen Vertragsstaaten anzuerkennen.

89 Ein Gewaltverhaltnıs kraft Gesetzes besteht immer dann, wenn die Sorgerechtsregelung unmittelbar und endgultıg aus dem Gesetz. zu entnehmen ist und ohne Dazwischentreten einer Bchorde oder eines Gerichts wirksam wird, so etwa nach der Stichentscheidsvorschrift aus Art. 263 des turk. ZGB, nach welcher 
lebendes Kind die Anerkennung dieses Gewaltverhältnisses vorschreibt. ${ }^{\circ 0}$ Dabei herrscht in Lehre und Rechtsprechung Uneinigkeit darüber, wie dieses Anerkennen zu bewerten ist. Dic herkömmlichen Interpretationsangebote zu Art. 3 MSA bringen in schematischer Weise entweder sofort die lex fori zur Geltung (Anerkennungstheorie) ${ }^{91}$ oder sic schließen im völligen Gegensatz dazu sogar die Zuständigkeit der deutschen Gerichte aus (Schrankentheorie). ${ }^{92}$ Eine kaum weniger formelhafte Theorie bestimnt die Zulässigkeit von Schutzmaßnahmen und damit auch dic Zuständigkeit deutscher Gerichte nach dem Heimatrecht, dies soll wiederum jedoch nur soweit gelten, wie die lex patriae Eingriffe in das Ex-lege-Verhältnis zuläßt. ${ }^{93}$ Über diese "regelungsfähige Lücke hinaus soll dann wicderum keine Zuständigkeit der deutschen Gerichte bestehen (Heimatrechtstheoric).

Der große Nachteil all dieser Theorien besteht darin, daß sie kaum in der Lage sind, die soziale Realität einer multikulturellen Gesellschaft mit einerseits erheblich divergierenden kulturellen Profilen bei den betroffenen Jugendlichen und andererseits dem Bedürfnis nach Andersleben bei den Migranten adäquat und flexibel zu erfassen. Daher legen es die sozial wissenschaftlichen und gesellschaftstheoretischen Erkenntnisse nahe, zu Art. 3 MSA eine Interpretation zu entwickcln, die flexibel die Bedürfnisse des Einzelfalls und insoweit die individuell sehr verschiedene Integration der Migrantenfamilien auffängt. Diesc Linie soll Respektierungstheorie genannt werden. Diese Interpretationsweise des Art. 3 MSA ist in Unterscheidung von den bisher dargelegten Theorien derjenige Lösungsansatz, der auf die Lebensformen der Migranten in der Bundesrepublik abstellt und damit das Grundanliegen des stärkeren, des wirklichen Respekts gegenüber den Rechtsordnungen der ethnischen Minderheiten ausdrückt. Bci der Begriffswahl ist nicht verkannt, daß sich auch die anderen Theorien in unterschiedlicher Form um Respekt vor dem Heimatrecht bemühen. Mit der Respektie-

der Mann bei Meınungsverschiedenheit zwischen shm und der Mutter in Erzichungsfragen immer das letzte Wort besitzt. Siehe auch die Sorgerechtsregelungen des iran. ZGB (Artt. 1169, I180), nach denen dem Vater zwıngend die elcerlıche Sorge uber das minderjahrige Kind zustcht. Die tatsachliche Personensorge steht der Mutter dabei nur bis zum 2. (Jungen), respektıve 7. Lebensjahr (Madehen) zu. Im deutschen Familienreche sieht $\$ 1$ yos BGB zur Zeit noch ein Ex-lege-Gewaltverhaltnis vor.

90 Das System der Konventıon, das Zustindıgkeit und anwendbares Reclat in enger Weise miteinander verbindet, weist Art. 3 MSA eine Doppelfunktion zu. Diese Norm schrankt nucht nur dic internationale Zustandigkeit eın, sondern sie setzt zugleich auch der an sich anwendbaren lex fori Grenzen. Die Bedeutung des Art. 3 MSA ist dabci das in der Praxis relevanteste und somit auch das am tiefsten diskutierte Rechtsproblem bet der Anwendung des Abkommens. Die Norm hat gleichsam eine Schlusselrolle fur das Verstindnis des MSA, denn an kemer Stelle wird die Anwendung des Aufenthaltsrechts starker durch Ex-lege-Gewaltverhaltnısse gehindert als hier, an keiner Stelle des MSA ist die Eindruchstelle fur auslandisches Recht großer als hier. Nach fast 2s Jahren, die das MSA in der Bundesrepublik in Kraft ist, sınd zu Art. 3 MSA die wichtigsten Fragen ausdiskutuert, jedoch ohne daß ein allgemeingultiger Konsens in Rechtsprechung und Lehre erzielt worden ware.

91 Diese Theorse ist am Vordringen begriffen und wird vor allem von der Literatur vertreten; siche etwa Staudinger/Kropholler, Vorbern. 208 ff. zu Art. 19 EGBGB; MunchKomm-Stehr, Art. 19 Anh. Rn. 110 , ${ }_{117}$ If.; Coester-Walcjen, ZBlJR 1990, 641, 645; Allinger (Fn. 87), S. 124; IPG 1972 Nr. 24 (Koln); IPG 1982 Nr. 1 4 (Hamburg); auch die Denkschrift der Bundesregierung zum MSA (BT-Drs. 6/947, S. I3) geht von der Anerkennungstheorie aus; vereinzelt schlossen sich desglexchen deutsche Gerichte dieser Interpretanionsweise an, so OLG Hamburg FamRZ 1972, 514; OLG Stuttgart NJW 1985, 566 und wohl auch AG Eschwege DAVorm $1995,389$.

92. Siehe nur BGH FamRZ 1973, 138; in der Folge dann auch BayObLG NJW 1974, 1092; OLG Hamm NJW 1975, 1083; OLG Karlsruhe NJW 1976, 485; AG Solingen FamRZ 1982, 738; die Theorie wird heute jedoch kaum noch vertreten.

93 Diese Theorie ward von der Rechtsprechung favorisiert; vgl. fur die deutsche Judikatur nur BGH FamRZ $1984,686,687$; BGH NJW-RR 1986, 1130 ; OLG Karlstuhe FamRZ 1995, 562; OLG Celle FamRZ 1989, 1324, 1325; $\Lambda$ G Kitzingen IPRax 1985, 298; unklar insowet OLG Frankfurt FamRZ 1995, 564, 565; vgl. wettere zahlreiche Nachweise beı MunchKornm-Siehr, Art. 19 Anh. Rn. 116 Fn. 136 sowie Boelck, Reformuberlegungen zum Haager Minderjahrigenschutzabkommen von 1961 (1994), S.9 Fn. 15; fur die osterreichische Rechtsprechung siche nur OGH IPRax 1992, 106; OGH IPRax 1993, 41 5: In der Literatur vertreten Palandt/Heldrich, Anh. zu Art. 24 EGBGB Rn. 25; Schurig, FamRZ 1975, 459, 462 ; Jayme, IPRax 1985,23 ; Rahm/Paetzold (Fn. 31), Rn. 448, die Heimatrechtstheorie; im Ergebus alunlıch Stocker, DAVorm 1975, 507, 511 . 
rungstheorie soll jedoch pointiert werden, daß die von den Migranten gelebte Rechtsform besonders ernst genommen werden soll. Die Respektierungstheorie mißt Art. 3 MSA dabei keine zuständigkeitsbeschränkende Wirkung bei. Daher sind in jedem Fall die Residenzbehörden zuständig, Maßnahmen zum Schutz des Minderjährigen zu treffen. Nach welchem Recht sich Umfang und Inhalt der Schutzmaßnahmen richtet, soll allerdings abhängig sein von der Einbindung und Verwurzelung des Minderjährigen in sein heimatliches Bezugsfeld, auf das es entscheidend ankommt. Danach findet das Heimatrecht des Kindes über Art. 3 MSA prinzipiell in den Fällen Berücksichtigung, in denen der ethnische Zusammenhang noch existiert und der Mindcrjährige in dieses heimatliche Umfeld integriert ist. Dies ist aus einer teleologischen Reduktion der Norm abzuleiten, die vor der Einwanderung in Furopa entstanden ist. Das Heimatrecht kann für dauerhaft Eingewanderte sinnvollerweise nur dann Geltung beanspruchen, wenn diese noch in ihrem ethnisch-sozialen Kontext leben. Insowcit bleibt das gesetzliche Gewaltverhältnis im Sinne des Art. 3 MSA bestehen, ${ }^{94}$ ist nur eventuell Korrekturen nach dem ordre public ausgesetzt.

Ist der ethnische Zusammenhang der Familie aufgebrochen und dokumentiert der Minderjährige durch sein Verhalten seine Assimilierungs- oder Syntheseleistung im Hinblick auf die Gesellschaft des Residenzstaates, führt die Respektierungstheorie dazu, daß die Anwendung der lex patriae auf den Minderjährigen nicht mehr angemessen ist. Denn in diesem Moment ist der vorbchaltlose, die Anwendung von Herkunftsrecht rechtfertigende Heimatbezug nicht mehr gegeben. Die Nähe zum Residenzstaat legt es dann nahe, den Minderjährigen nach der lex fori zu behandeln. So ermöglicht es die Respektierungstheoric den Familien aus ethnischen Minderheiten, dann nach ihrem Heimatrecht behandelt zu werden, wenn das sie umgebende kulturelle Umfeld dies nahclegt, und sie ermöglicht weiter, die mitunter sehr verschiedene Integration der einzclnen Familien zu berücksichtigen. Dic Respektierungstheorie vereinfacht mithin die Anwendung des Abkommens erhcblich.

Als Gegenstück zu dem crhöhten Respekt für die lex patriae auf der Ebene des anwendbaren Rechts muß - sozusagen als Kehrscite derselben Medaille - noch die Möglichkeit bestehen, auf der individuell-konkreten Stufe des jeweiligen Einzelfalls das Heimatrecht auszuschaiten. Dies ist nach der hier vorgeschlagenen Lösung die zweite Prüfungsstufe des fremden Rechts und gleichzeitig der Einsatzmoment für die nationale Vorbehaltsklausel des ordre public, die dann das fremde Recht ausschließt, falls die Anwendung zu einem Ergebnis führt, das gegen grundlegende Prinzipien der deutschen Rechtsordnung verstößt." Ds Doch auch bei Heranziehung des ordre public nach Art. 6 EGBGB muß ein sorgsamer Umgang mit dem Heimatbezug der Migranten eingcübt werden. Dabei wären Zugeständnisse an die Rechts- und Wertvorstellungen der Einwanderer durch inhaltliche Relativierung des ordre public möglich. Es muß also danach gefragt werden, ob das Anwendungsergebnis ausländischen Rechts von der deutschen öffentlichen Ordnung eklatant abweicht und als solches für die deutschen Anschauungen von Recht und Sitte schlechterdings untragbar ist. ${ }^{96}$

\footnotetext{
94 Ein ahnlıcher Ansatz findet sich bei Allinger (Fn. 87), S. 78 f. als matcriell-rechtliche Theorie beschrieben.

95 Im nationalen IPR ist der ordre public in Art. 6 EGBGB geregelt, vgl. dazu Palandt/Heldrich, Art. 6 EGBGB Rn. Iff.; MunchKomm-Sonnenberger, Art. 6 Rn. Iff.; eine inhalclıch gleıche Klausel enthàlt auch Art. 16 MSA, vgl. dazu Staudinger/Kropholler, Vorbem. 65 zu Art. 19 EGBGB. Dabei konkreti. siert vor allem Art. 6 Satz 2 EGBGB einen wichtigen, mittlerweile den bedeutendsten Bereich des Inhalts der w wesentlichen Grundsaicze des deutsehen Rechts" durch dic explizite Erweiterung des Prufungsmaßstabes um die Grundrechte. Diese fanden mit Inkrafttreten des IPR-Neuregelungsgesetzes vom 1.9. 1986 (BGBl. $1986 \mathrm{I}$, S. 1142) Aufnahme in die allgemeine Ordrc-public-Klausel und hiermit in den Schuczbereich der offenthchen Ordnung.

$96 \mathrm{Vgl}$. auch MünchKomm-Sonnenberger, Arr. 6 Rn. 71 ; BGHZ 50, 370, 375 f.
} 
Dabei verneint die hM ${ }^{97}$ im Rahmen des Art. 6 Satz 2 EGBGB eine inhaltliche Relativität. Anders als bei Satz 1 der Norm hat der Gesetzgeber in Satz 2 darauf verzichtet, daß die Unvereinbarkeit des fremden Rechts mit den Grundrechten »offensichtlich «, also von besonderer Schwere sein muß.$^{98}$ Demnach soll die Eliminierung des fremden Rechts bei Grundrechtsverletzungen nicht auf besonders unerträgliche Verstöße begrenzt werden können, ${ }^{99}$ vielmehr dürfe nicht zwischen tragbaren und untragbaren Grundrechtsverstößen unterschieden werden. ${ }^{10}$ Satz 2 wird somit in der Weise interpretiert, daß jede durch Anwendung ausländischen Rechts in concreto bewirkte Grundrechtsverletzung ein Eingreifen des ordre public auslöst. ${ }^{101} \mathrm{Die}$ sachliche Rechtfertigung für die fehlende inhaltliche Relativität des ordre public bei Grundrechtsverstößen wird damit begründet, daß Verletzungen der Grundrechte als "oberste Lcitprinzipien ${ }^{102}$ stets (inhaltlich) unrelativierbar seien. ${ }^{103}$

Nun ist aber zu überlegen, ob die apodiktisch-puristische Vorstellung haltbar ist, jeder Grundrechtsverstoß habe automatisch die Unanwendbarkeit der ausländischen Norm zur Folge. ${ }^{1 \cdots}$ Der Ausgangspunkt der Überlegungen dazu muß sein, daß eine Differenzierung der Verfassungseinwirkung auf das ausländische Recht unstreitig vonnöten ist; dies bestätigt auch die hM, wenn sie die Verfassungseinwirkung über die örtliche Relativität differenzieren will. ${ }^{105}$ Die Erwägungen zum Zusammenleben in einer multikulturellen Gesellschaft legen aber gerade einc inhaltliche Relativierung, wie sie in Satz I der Vorbchaltsklausel statuiert ist, auch für Art. 6 Satz 2 EGBGB nahe. Dies folgt aus der prinzipiellen Erwägung, Migranten eine Form von kollektiven Rechten und kollektiver Anerkennung zuteil werden zu lassen, sowie aus

97 MunchKomm-Sonnenberger, Art. 6 Rn. 38; Spickhoff, JZ 1991, 323, 324; Henrich, RabelsZ 1972, 2, 4 ff.

99 Spickhoff, JZ 199r, 323, 324; BGH F.mRz 1993, 316,317; vgl. d.xzu auch die Regierungsbegrundung in BT-Drs. IC/504, S. 44: "Eine Grundrechrsverleczung im Einzclfall durch Anwendung einer Vorschrift fremden Ruchts ist daher als unvereinbar mit dem deutschen ordre public stets von vornherein ausgeschlossen. Sit' ware im Sinne des Sarzes i immer ein roltensichtlicher, Verstoß gegen wesentliche Grundsàtze des deutschen Rechts. Diese stellt die von Satz I unabhäng:ge Formulierung der Voraussetzung fur die Nichsanwendung fremden Rechts in Sacz 2 sicher. \&

99 Henrich, Rabels $L$ 1972, 2, 4ff; dies hatu der BCiH (FamRZ 1973, 138, 442) allerdings in seiner ersten Entscheidung nach den Spanierbeschluß ubersehen, als er untragbare von tragharen Grundrechtsverstößen unterscheiden wollte.

100 So schon BVerfG FamRZ 1971, 414, 421; siehe auch Kropholler, IPR (1994), \$36 IV I Fn. 23; Berger LM Nr. 3 (zu Art. 6 EGBGB 1986).

I01 Vgl. BVerfG FamRZ 1971, 414, 421; MunchKomm-Sonnenberger, Art. 6 Rn. 38; in diesem Sinne auch Henrich, FamRZ $1974,105,110$.

102 Spickhoff, JZ 1993, $210,212$.

$\mathrm{IO}_{3}$ In diesem Sinne auch die Regicrungsbegrundung in BT-Drs. 10/504, S. 44

${ }_{104}$ Ablehnend anscheinend Luderitz, IPR (1992), Rn. 210.

10s Bei der ortlichen Relativitat oder Inlandsbeziehung handelt es sich um eın Kriterium, das die Anwendung des deutschen ordre public dann ausschlıßen will, wenn die Migrantenfamilien nicht genug Bindungen an den Aufenthaltsstaat geknüpft haben. Diese sollen festgestellt werden durch Bestimmungsfaktoren wie dem gewohnlichen Aufenthalt, der Staatsangehorigkeit sowie der kulturellen und rechtlichen Bındung an das Inland, vgl. Staudinger/Blumenwitz, Art. 6 EGBGB nF Rn. $114 \mathrm{f}$. sowie MunchKomm-Sonnenberger, Art. 6 Rn. 74. Der Inlandsbezug erscheint aber nicht als die geeignete Stufe, um die Einwirkung der deutschen Grundrechte auf die Migrantenfamilien zu relacivieren. Dies ist damit zu begrunden, daß eıner solchen Familie, wenn sie schon lange Jahre in der Bundesrepublik lebt, die Inlandsbeziehung nicht schlechterdings unter dem Hinweis auf ein Leben nach den heimischen Traditionen des jeweiligen Heimatstaates auch in der Bundesrepublik abgesprochen werden kann (so aber das OLG Saarbrucken FamRZ 1992, 848, 849 als Vorinstanz zu BGH FamRZ 1993, 316). Dies ignoriert dıe vorhandenen Bındungen an den Residenzstaat, dıe regelmäßıg bei Mıgrantenfamılien exıstieren, selbst wenn diese im ethnischen Verbund leben. Es erscheint nicht angemessen, den Migrantenfamilien, die sich oftmals auf der agleitenden Skala zwischen ffemd und sdeutsch»a bewegen (Coester, IPRax 1991, 236), bestimmte Rechtsstandards des deutschen Rechtskreises mit Bezug auf den Binnenbezug vorzuenthalten Dies entpuppt sich zu sehr als nur scheinbarer Respekt vor der fremden Rechtsordnung, der eher eine Verdeckung des Konflikts beinhaltet und eine Art Gleichgultigkeit gegenuber den Geschehnissen in der ethnischen Gemeinschaft ausdruckt. So muß die ortliche Relativitat vielmehr als Basis betrachtet werden, von welcher aus die Úberlegungen z.u emer solchen Grundrechtsrelativierung anzustellen sind. 
der Tatsache, daß es für die Migrantenfamilien und vor allem für das Kind sehr häufig einen engen Bezug zur eigenen Ethnie gibt, der charakterisiert ist durch Freizeitverhalten, familiäre und außerfamiliäre Prägung, durch Sprache und Religion, eben durch das Leben in der ethnischen Community. Dieser Heimatbezug entspricht dann jenem bestimmten Zusammenhang, in dem nach der Politik der Differenz die Zubilligung von Sonder- und Gruppenrechten für die Migranten gerechtfertigt ist. Somit empfiehlt es sich, ihnen zu gestatten, ihre Angelegenheiten bis zu einer bestimmten Grenze ohne Überwölbung durch die deutschen Grundrechte zu regeln. Bei Sachverhalten mit Auslandsberührung kommen die deutschen Grundrechte also nicht ohne jede Einschränkung zum Zuge.

Als Modell einer differenzierten Grundrechrscinwirkung bietet es sich an, dic Politik der Differenz hier auch rechtlich umzusetzen. Im Sinne der Erwägungen, nach denen der Staat in multiethnischen Gesellschaften nicht streng neutral ist, sondern sich für das Bestehen bestimmter Ethnien und Kulturen einsetzt und so gewisse Grundrechte in einem bestimmten Zusammenhang anders interpretiert werden können als in einem anderen, ist hier daran zu denken, die ausländischen Familien nur auf einen elementaren Grundrechtsbereich unrelativierbar zu verpflichten, während ihnen in einem peripheren Raum gestattet wird, ihre Verhältnisse grundrechtsautonom zu gestalten. Dieser periphere Bereich ist dann der Ort, an welchem die Unterstellung der Migrantenfamilie unter die deutschen Grundrechte zurückstehen muß gegenüber dem Anspruch, die Wichtigkeit des kulturellen Überlebens einer ethnischen Minderheit zu sichern. Insoweit sind "grundrechtseinschränkende Statusgarantien ${ }^{106}$ zu gewährleisten. Hier ist die Schranke für den Grundrechtsuniversalismus. Damit wird gleichzeitig die Frage aufgeworfen, wie der elementare Grundrechtsbereich vom peripheren abgegrenzt werden kann. Hier müssen die als überethnisch und somit in einer multikulturellen Gesellschaft verbindlich bezeichneten Werte diesem elementaren Grundrechtsbereich entsprechen. So zählt zu den unrelativierbaren Grundrechten das Recht auf Leben, die körperliche Unversehrthcit und ctwa das Diskriminierungsverbot. Der periphere Bereich beschreibt auf der anderen Seite das juristische Pendant zu den Werten, die in einer multikulturellen Einwanderungsgesellschaft nicht unbedingt absolut zu schützen sind. Dics bedeutet im Ergebnis, daß für die hier interessierenden Kontroversen insbesondere die Kindesgrundrechte, aber auch die Rechte der Frau aus Art. 3 II GG einer differenzierten Betrachtung unterliegen können. ${ }^{107}$

Rechtsdogmatisch ist diese inhaltliche Differenzierung in der Wcise zu konstruieren, daß Art. 6 Satz 2 EGBGB teleologisch auf Verstöße im grundrechtlichen Elcmentarbereich beschränkt wird. Insoweit ist dann tatsächlich jeder Grundrechtsverstoß ein Interventionsgrund. Dann muß Art. 6 Satz I EGBGB systematisch so gelesen werden, daß er die peripheren Verletzungen von Grundrechten erfaßt, aber nur offensichtliche Verstöße gegen den ordre public sanktioniert. In diescr Weise ist im Ergebnis ein umfassender Grundrechtsoktroi gegenüber den Ausländern abzuwenden, glcichwohl aber gesichert, daß die elementaren Werte der deutschen Rechtsordnung genügend Berücksichtigung finden. Insofern ist nach dieser Lösung entgegen der hM eine inhaltliche Relativität auch für Satz 2 von Art. 6 EGBGB anzunehmen. Anhang einiger ausgewählter Beispielsfälle sei die Wirkung der inhaltlichen Relativität illustriert.

106 So der Terminus von Habermas (Fn. 3), S. 1 52.

107 Diese differenzıerte Betrachtung, die eınen notwendigen Kompromiß zwıschen der Forderung multıkultureller Toleranz und dem Eintreten gegen patriarchalische (Rechts-) Vorstellungen erfordert, wird in Zukunft weiter nuanciert werden mussen. Grundsatzlich bietet der ordre public eine gute Handhabe fur diese Aufgabe. 
Ist über ein absolures Umgangsverbot eines türkischen Vaters zu befinden, das dieser gegenüber seiner Tochter verhängt hat, oder stcht eine Umgangsbeschränkung in der Weise in Rede, daß der Vater oder ein von ihm beauftragter Sohn bei abendlichen Treffen der Tochter mit gleichaltrigen jugendlichen Männern anwesend sein will, ${ }^{108}$ und wird dies durch das türkische Recht im Rahmen der elterlichen Gewalt gedeckt, so ist zunächst auf der materiell-rechtlichen Ebenc nach der Respektierungstheorie zu klären, ob aufgrund intakten ethnischen Verbundes auf den Konflikt generellabstrakt die lex patriae anzuwenden ist. Wenn ein solcher Verbund gegeben ist, ist auf der individuell-konkreten Stufe zu prüfen, ob diese Einschränkung der sozialen Kontakte der Tochter durch den türkischen Vater, die in einem vergleichbaren deutschen Fall als ein Verstoß gegen $\$$ I 666 BGB eingestuft werden müßte, bei türkischen Familien gegen den ordre public verstößt. Im Ergebnis ist anzunehmen, daß in einem solchen Fall der elementare Bereich des Kindeswohlgrundrechts noch nicht berührt ist. Hier muß die Gleichbehandlung des türkischen Kindes zurückstehen zugunsten des Rechts der Eltern oder der Familie, ihre Konflikte nach den eigenen Rechts- und Moralvorstcllungen zu regulieren. Eine solche Einschränkung der sozialen Kontakte des Kindes tangiert nach der Politik der Differenz keine der unverbrüchlich garantierten Grundfreiheiten in einer multikulturellen Gesellschaft.

$\mathrm{Zu}$ überlegen ist weiterhin, wie es zu beurteilen ist, wenn die türkischen Eltern ihrer Tochter verbieten, einc Ausbildung mit uneingeschränkten Kontakten zum anderen Geschlecht zu absolvieren. ${ }^{109}$ Verstößt dieses Verbot gegen ein unrelativierbar zu garantierendes Grundrecht? Hier handelt cs sich um cinen Grenzfall, an dem die Durchsetzung von differenz-politisch relevanten elterlichen Moral- und Verantwortungsvorstellungen gegen Kindes- und Gleichberechtigungsinteressen einzugrenzen ist. Streng genommen gehört das Recht auf optimalc Ausbildung nicht zu den uneingeschränkt zu schützenden Rechten in einer multikulturellen Gescllschaft, insbesondere wenn die Ausbildung noch nach Erreichung der Volljährigkeit absolviert werden kann. Eine Ausbildungsbeschränkung verstößt jedenfalls dann gegen elementare Grundsätze des Art. 3 II GG und somit gegen den ordre public, wenn sie auf der Abschottung von Frauen beruht.

Wie wirkt nun die inhaltliche Relativität, wenn es darum geht, im Rahmen von

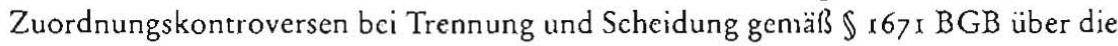
Anwendung eines ex lege bestehenden ausländischen Gewaltverhältnisses zu befinden? Dies sei an einem der leitenden BGH-Entscheidung ${ }^{\text {to }}$ nachempfundenen Fall illustriert: Hier ging es um die Regelung der elterlichen Sorge nach der Scheidung eines in Deutschland lebenden iranischen Paares. Aus der Ehe waren zwei Mädchen und ein Junge hervorgegangen. Auch hier kam im Ausgangspunkt die lex patriae zum Zuge, weil es sich um eine rein iranische Familie handelte, so daß das deutschiranische Niedcrlassungsabkommen von 1929 (NA) anwendbar war. "' Nun war zu untersuchen, ob die Anwendung der lex patriae und somit die Beachtung des Exlege-Gewaltverhältnisses in concreto den elementaren Bereich dor Kindergrundrechte, des Kindeswoh]s berührte.

Nach dem Differenzmodell ist es hier vorstellbar, die staatliche Interventionsschwelle bei der Sorgcrechtsverteilung nach Scheidung zu erhöhen. Danach würde dic gerichtliche Rcgelungsbefugnis erst dann cinsetzen, wenn dic Ex-lege-Sorge-

$108 \mathrm{Vgl}$. einen solchen Fall in LG Berlin FamRZ 1983, 947; zu der elterlichen Gewalt im turkischen Recht siehe Serozan, ZBIJR 1991, 492, 492 .

$109 \mathrm{Vgl}$. dazu nur die Sachverhalte LG Berlin FamRZ 1983, 947; KG IPRax 1985, 347

iso BGH FamRZ 1993, 316.

11 Abgedruckt in RGBI. 1930 II, S. 1002, 1006; Bekanntmachung uber dic Weitergeltung im BGBI. 1955, II, S. 829; das Abkommen bringt von vornherein zwingend das Heimatrecht zur Anwendung. 
rechtszuweisung nach fremdem Recht die Schwelle der Kindeswoblgefährdung errcichen würde."12 Dies läßt sich danit begründen, daß erst dann die elementaren Kindesgrundrechte berührt sind. Die Regelungsbefugnis des Gerichts in Rahmen des ordre public ist danach noch nicht gegeben, wenn dic Sorgcrechtsregelung nicht in vollkommener Weise den deutschen Vorstellungen entspricht.. ${ }^{13}$

So kann es dazu kommen, daß die (deutschen) Vorstellungen von optimaler Plazierung des Kindes zurückstehen müssen hinter denienigen Ideen von angemessener Regelung des Sorgerechts und adäquater Zuteilung des Kindes, die in den Ex-legeGewaltverhältnissen der ausländischen Rechtsordnungen zum Ausdruck kommen. Innerhalb des ethnischen Verbunds ist also erst dann Raum für Ordre-public-Eingriffe, wenn die ex lege vorzunehmende Zuweisung des Sorgerechts das Kindeswohl gefährdet. Darüber hinaus verbleibt kein Anwendungsspielraum für die Korrektivklausel in der Community. Insofern hat in der multikulturellen Gesellschaft das Fortbestehen des ethnischen Kollektivs an diesem Punkt Vorrang vor der Zuweisung nach deutsch-geprägten Kindeswohlgrundsätzen; insofern werden die über dic Formel des Kindeswohls zum Tragen kommenden Kindesgrundrechte hier inhaltlich relativiert. So könnte mithin im Ergebnis des Beispielsfalls das den Vater ex lege begünstigende Sorgerecht auch ordre-public-konform bestehen bleiben, falls das Wohl des Kindes dadurch nicht gefährdet wird und hinter ihm cin adäquater Familienzusammenhang stcht." ${ }^{14}$ Dagegen hat der BGH das väterliche Sorgerecht nach der ausländischen Rechtsordnung nach Art. 6 EGBGB korrigiert und dem Vater lcdiglich das Recht der Vermögenssorge für das vermögenslose Kind übertragen. Damit wird der Respekt vor der ausländischen Rechtsordnung zum bloßen Schein.

Die inhaltliche Relativierung bietet aber nicht nur bezüglich der Kindesinteressen einc Lösung an. Auch zur Diftercnzierung eincs Ordrc-public-Verstoßes hinsichtlich der elterlichen Gleichberechtigung bei der Sorgerechtsverteilung eignet sich dieses Modell. Dies sei an einem Fall verdeutlicht, den das OLG Düsseldorf zu entscheiden hatte."' Eine deutsche Mutter und ein jordanischer Vater stritten um das Soryerecht für ein Kind, das sich im Heimatstaat des Vaters aufhielt. Rechtlich ging es dabei um die Ordre-public-Relevanz von Art. Iss des jordanischen Personenstandsgesetzes, der grundsätzlich die nicht-moslemischen Frauen vom Sorgerecht ausschließt.

Hier wird wohl ein Verstoß gegen den elcmentaren Bereich des Grundsatzes der Gleichberechtigung angenommen werden müssen. ${ }^{16}$ Ein so umfassend religiös begründeter Ausschluß vom Sorgerecht durch die Ex-lege-Zuweisung verstößt nicht nur gegen Arr. 3 II GG, sondern verletzt darüber hinaus das Elternrecht der Mutter, da sie jedweder Chance beraubt wird, ihre Rechte wahrzunehmen und ihre Pflichten zu erfüllen. Eine solche Vorenthaltung von Rechten, die einer Diskriminierung gleicht, kann auch in einer multiethnischen Gescllschaft nicht gewährt werden. Hinzu komme noch, daß ein solcher komplettcr Sorgerechts-Ausschluß der Mutter auch mit einem Verstoß gegen die elementaren Kinderrechte einhergchen wird. Denn die Mutter von der elterlichen Sorge auszuschließen, weil sie der falschen Religion angehört, gefährdet von Anfang an das Kindeswohl und verletzt somit auch dessen elementare Rechte.

112 Dabei ist auch der Willen des Kındes zu berucksichtigen, das gemaß $\S$ so b FGG beı der Sorgerechtszuteilung anzuhoren ist.

113 So wohl auch Rauscher, JR $1994,184,188$

114 Eine Kindeswohlgefahrdung ware denkbar bei hoher Arbeitsbelastung des Vaters in der Bundesrepublik.

115 OLG Dusseldorf NJW-RR 1994, 7.

116 So in Ergebnis auch das OLG Dusseldorf (NJW-RR 1994, 7, 8) 
Der Vorbehalt des ordre public und somit der Einsatz der Korrektivklausel des Art. 6 EGBGB und der deckungsglcichen staatsvertraglichen Klauseln ist in den vergangcnen 25 Jahren umfassend ausgedehnt worden. In den Mittelpunkt sind verstärkt die Kindesinteressen gerückt, die dabei eine vertassungsrechtliche Absichcrung erfahren haben. Zudem ist dic zurückhaltende Anwendung des ordre public auf ausländische Familien aufgegeben worden. Die Vorbehaltsklausel wird nun extensiver angewendet, und Migrantenfamilien werden den gleichen Kriterion unterworfen wie die deutschen Familien. Eine Differenzierung in der Verfassungseinwirkung will die hM nur durch einc örtliche Relativicrung vornehmen, die jedoch bei dauerhafter Einwanderung eine Korrektur des Heimatrechts nicht trägt. In Wirklichkcit geht es um cine inhaltliche Relativicrung, wie sie nach dem sozialwissenschaftlichen und gesellschaftstheoretischen Befund crforderlich ist. Die "deutsche öffentliche Ordnung " ist nicht der Ort, an dem sich die Rechtsstandards der deutschen Rechtsordnung uneingeschränkt durchsetzen können. Migrantenfamilien sind nicht rigide den rechtlichen Bedingungen des Residenzstaates zu unterwerfen. Thnen ist die Chance auf Bewahrung kultureller Besonderheiten auch über das internationale Familienrecht zu erhalten. Auf der anderen Seite ist auch denjenigen, die sich vollständig assimilieren wollen, die Möglichkeit dazu einzuräumen. Dies ist mit dem hier vorgestellten Modell möglich.

\section{Markus Klemmer \\ Gesetzesbindung und Richterfreiheit}

Die Entscheidungen des Reichsgerichts in Zivilsachen während der Weimarer Republik und im späten Kaiserreich

Die Weimarer Republik gilt auch hinsichtlich der Entwicklung der Haltung der Richterschaft zum gcschriebenen Gesetz als Zeitenwende. Gerade die Nachkriegszeit mit ihren Inflationswirren stellte die Rechtsprechung vor noch nie dagewesene Konflikte. Die Monographie berücksichtigt über 600 Entscheidungen und eine Vielzahl von zeitgenössischen Stellungnahmen von Reichsgerichtsräten, deren Äußerungen erstmals vor dem Hintergrund der von ihnen gefällten Urteile untersucht werden.

Der Verfasser zieht eine ernüchternde Bilanz hinsichtlich des vielgerühmten "Befreiungswerkes" der Weimarer Richterschaft. Er zeigt auf, daß die bisherige Forschung sich vom Krisenmanagement, welches die Rechtsprechung in der kurzen Zeit zwischen 1918 und 1923 teilweise betreibt, zu weitreichenden Aussagen über eine generelle Richterfreiheit hat bewegen lassen, die so nicht haltbar sind.

Der Verfasser ist als Rechtsanwalt tätig.

1996, $496 \mathrm{~S}$., brosch, $124,-D M, 905,-\ddot{o} S, 110,50 \mathrm{sFr}$, ISBN 3-7890-4259-5

(Fundamenta Juridica, Bd. 30)

NOMOS Verlagsgesellschaft 76520 Baden-Baden 